\title{
Modelling Social-Aware Forwarding in Opportunistic Networks
}

\author{
Chiara Boldrini, Marco Conti, and Andrea Passarella \\ IIT-CNR, \\ Via G. Moruzzi 1, 56124 Pisa, Italy \\ \{chiara.boldrini, marco.conti, andrea.passarella\}@iit.cnr.it
}

\begin{abstract}
Opportunistic networks are one of the most promising evolutions of the traditional Mobile Ad Hoc Networks paradigm. Communications in an opportunistic network rely on the mobility of the users: each message is handed over from node to node, making hop-by-hop decisions to select the node that is better suited for bringing the message closer to its destination. Algorithms exploiting social-awareness are emerging as one of the most efficient categories of forwarding algorithms. However we are currently lacking analytical models able to characterize the performance of social-aware forwarding in opportunistic networks. In this paper we start to fill this gap by proposing an analytical model for the expected number of hops and the expected delay experienced by messages when delivered in an opportunistic social-aware fashion. The model is then used to characterize how the expected delay experienced by messages varies with the different social structures in the network of the users.
\end{abstract}

Keywords: opportunistic networks, forwarding protocols, socialawareness, analytical model.

\section{Introduction}

In the broad area of wireless multi-hop networking, Delay Tolerant Networks (DTNs) have recently stood out because of their ability to enable communications even when protocols designed for traditional Mobile Ad Hoc Networks (MANET) cannot do so. In fact, the main requirement of MANET protocols, i.e., the presence of an end-to-end path connecting the source and the destination of a message, can be rarely satisfied in networks, e.g., made up of subnetworks connected only by satellite links [4, or where the nodes are people moving around with their hand-held devices [16. The latter case is the scenario considered in this paper. In order to differentiate between the different applications of the delay tolerant paradigm, such networks have been named Pocket Switched Networks (PNSs) or opportunistic networks, because they opportunistically exploit contacts between users.

Messages in PSNs are routed along a multi-hop path across the nodes of the network. Being PSNs so unstable, source routing is inapplicable as the route 
chosen by the source of the message is likely to change within a short time. For this reason, forwarding decisions in opportunistic networks are made hop by hop. The key problem of message forwarding in PSNs is thus the selection of the node to which the message (or a copy of the message, in the case of multi-copy schemes) should be handed over. First and simplest implementations of this new communication paradigm involved a great number of copies of the same message to be spread across the network, in order to maximize the probability that one of them will eventually arrive at the destination [21. Smarter strategies have been developed later, with the aim of selecting only the best relays as next hops for each message. In particular, social-aware strategies have proven [211] to be very effective in forwarding messages in an opportunistic network. Their main idea is that, while the connectivity graph of the network might be extremely unstable, the social graph, i.e., the network of relationships between users, is expected to vary on a much larger timescale than that typically of interest for the delivery of messages. This approach is indeed effective because of the correlation between sociality and mobility [17]: knowing social relationships between users enables us to estimate the likelihood of future encounters between nodes, which represent a forwarding opportunity.

Despite being so popular as forwarding strategies, social-aware schemes are typically difficult to model analytically. The main contribution of this paper lies in the definition of an analytical model for the evaluation of social-aware singlecopy forwarding schemes. This model, based on Markov Chains, allow us to describe a way for computing significant quantities, such as the expected number of hops or the expected delay, that characterize the forwarding performance.

The paper is structured as follows. In Section 2 we review the state of the art on forwarding modelling for opportunistic networks. In Section 3 we describe our analytical model for social-oblivious and social-aware forwarding. In Section 4 we use the above model for evaluating the performance of four reference forwarding strategies with different underlying social structures for the network of the users. Finally, in Section 5 we conclude the paper.

\section{Related Work}

As anticipated in the previous section, forwarding protocols can be classified, according to the type of information that they exploit when making forwarding decisions, into social-oblivious and social-aware protocols. Social-oblivious protocols do not use at all information on the way nodes meet or relate with each other. This is the case of the Epidemic protocol [21], whose strategy is to generate and hand over a new copy of the message to each node encountered, and of the Direct Transmission protocol [9], in which messages can only be delivered to the destination when encountered directly. The performance of these protocols is typically poor because either they consume a lot of resources and overload the network (Epidemic) or they are not able to find a path to the destination even when many are available (Direct Transmission). For this reason, they are typically used as a baseline for performance evaluation. 
Social-aware protocols, instead, exploit the social structure of the network of users in order to make forwarding decisions. This is because social-awareness enables the prediction of user encounters, which constitute forwarding opportunities. Some social-aware schemes focus only on encounters between nodes. This is the case of PROPHET [13, where the delivery probability of a node for a given destination is estimated based on previous encounters between nodes. Another approach is based on the exploitation of the roles of the nodes in the social graph associated with the network of users. The main idea is that nodes that are more central in the social graph are likely to be better forwarders than the other nodes. Bubble Rap [11] and SimBet [5] belong to this category. Social context-aware protocols keep track of a variety of information on the environment - context - the users live in (e.g., the people they meet, the friends they have, the places they visit). Context information is then used to quantify the ability of nodes to deliver messages. The HiBOp [2] protocol pertains to this group.

As far as modelling is concerned, quite a few frameworks have been proposed for social-oblivious forwarding schemes 22210|8|18 19]. Epidemic models, Markov Chains and random walk on graph are the mathematical tools used to model important metrics such as the expected delay. The problem with these model is that they all consider homogeneous networks, i.e., networks where node movements are independent and identically distributed. This is not the case of real networks made up from human users moving with their portable devices: some users may cluster and move together, others may never get in touch with each other. Such heterogeneousness has been so far considered only in 20]. However, authors of 20] focus on multi-copy schemes, while in the following we consider single-copy schemes, i.e., schemes in which there is at any time just one copy of the message to be delivered.

\section{A Semi-Markov Model for Message Forwarding}

In this section we model the forwarding process as a semi-Markov process, and then we perform a transient study in order to compute the expected number of hops and the expected delay experienced by messages. We start with a general framework, which we then specialize for four forwarding protocols representative of different approaches to forwarding. Let us first introduce in the next section the network model that we consider.

\subsection{Network Model}

Our model considers a network with $N$ nodes, moving around and meeting with each other. During contacts, nodes can exchange messages. For the sake of simplicity, we hereafter assume that messages can be exchanged only at the beginning of a contact between a pair of nodes (i.e., no periodic probing for new messages to relay during long contact periods), and that the transmission of the relayed messages can be always completed within the duration of a contact. The latter assumption is also justified by the fact that given the high dynamics of 
an opportunistic networks the file size is expected too be small [14. In addition, we assume that each message is a bundle [6], an atomic unit that cannot be fragmented 11 We also assume infinite buffer space on nodes. Given that we are considering single-copy schemes, buffer size is not expected to be critical, at least from low to medium network load. All the above assumptions allow us to isolate, and thus focus on, the effects of node mobility from other effects.

Given that messages are handed over from node to node before reaching their destination, the way nodes move heavily affects the delay experienced by messages. As for the mobility, the main role in the experienced delay is played by the inter-meeting time, which is defined as the time between two consecutive meetings between the same pair of nodes. In this paper we assume that such inter-meeting times can be described with an exponential distribution. Characteristic mobility times have been shown to follow an exponential distribution at least in their tail [12] 77. Trading accuracy for tractability, here we assume the exponential property for the entire distribution. As a future work, we plan to relax the exponential assumption. In the following we denote as $\lambda_{i j}$ the rate of the exponential distribution describing the process of encounters between two nodes $i$ and $j$.

\subsection{Reference Forwarding Strategies}

We generalize the variety of protocols described in Section 2 into the two main categories of social-oblivious and social-aware forwarding protocols. For these categories, we consider the following policies, which identify important traits of existing forwarding strategies. More specifically, among the social-oblivious schemes we consider the following.

Definition 1 (Direct Transmission). The source node can only deliver the message to the destination itself.

Definition 2 (Always Forward). The source node hands over the message to the first node encountered, and so does each intermediate node. The process stops when the message is delivered to the destination.

As for the social-aware schemes, a message (be it on the source node or on an intermediate relay) is handed over to another node only if the latter has a higher probability (we call it fitness) of bringing the message closer to its destination than the node currently holding the message. Based on how the fitness is computed, we define the following two policies.

Definition 3 (Direct Acquaintance). The source and each intermediate relay hand over the message to the first encounter having a higher fitness, where the

\footnotetext{
${ }^{1}$ Fragmentation can indeed add additional delay at the destination or, even worse, impair communication at all when some fragments are lost, due to the high round trip time of opportunistic networks.
} 
fitness $F^{D A}$ is defined as the frequency of a direct meeting with the destination (Equation 1).

$$
F_{i, d}^{D A}=\lambda_{i, d}, \forall i \neq d
$$

Definition 4 (Social Forwarding). Messages are delivered through a path with positive gradient of fitness, where the fitness $F_{i, d}^{S F}$ of node $i$ for a message addressed to node $d$ is computed (Equation 2) as the weighted sum of the fitness for a direct acquaintance $\left(F_{i, d}^{D A}\right)$ and the fitness for an indirect meeting $\left(F_{i, d}^{I}\right)$.

$$
F_{i, d}^{S F}=\alpha F_{i, d}^{D A}+(1-\alpha) F_{i, d}^{I}, \quad \text { where } 0<\alpha<1
$$

Component $F_{i, d}^{D A}$ is defined as in Equation 1. The second component is a measure of the likelihood of encountering a node that has high delivery probability for the destination and it is defined according to the following:

$$
F_{i, d}^{I}=f\left(F_{j, d}^{D A}\right) \quad \forall j \mid \lambda_{i j} \neq 0, j \neq d .
$$

There is a variety of possible choices for function $f$ in Equation 3. Without loss of generality, in the rest of the paper we use $f \equiv \max (\cdot)$.

Differently from the Direct Acquaintance policy, the Social Forwarding strategy is able to detect not only direct meetings with the destination, but also meetings with people that have a high probability of delivering the message to the destination. This strategy enables the exploitation of the delivery skills that are present in the environment surrounding the users, and not only of those of the user itself. In Section 4.2 we will show how important can be this exploitation.

\subsection{The Forwarding Process as a Semi-Markov Process}

A semi-Markov process is one that changes states in accordance with a Markov chain (called embedded or jump chain) but where transitions between states can take a random amount of time [15. As such, it is fully described by the transition matrix associated with its embedded chain and by $T_{i}^{e x i t}, \forall i=0, \cdots, n$, where $T_{i}^{\text {exit }}$ denotes the distribution of the time that the semi-Markov process spends in state $i$ before making a transition.

We express our semi-Markov process in terms of the embedded Markov chain in Figure 1. Assuming that node $i$ is currently holding a message whose

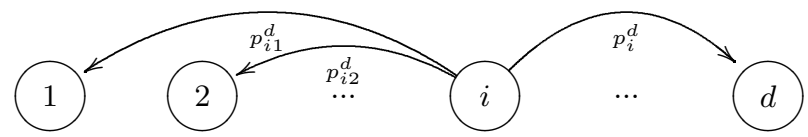

Fig. 1. Fragment of the embedded Markov Chain (valid for all $i \neq d$ ) 
destination 2 is $d$, the probability $p_{i j}^{d}$ that node $i$ will delegate the forwarding of the message to another node $j$ is a function of both the likelihood of meeting node $j$ and the probability that node $i$ will hand over the message to node $j$ according to the forwarding policy in use.

Theorem 1 proves that, under the exponential assumption for inter-meeting times (see Section 3.1), the semi-Markov process that describes the forwarding evolution becomes a Continuous Time Markov process, in which $T_{i}^{\text {exit }}$ follows an exponential distribution.

Theorem 1 (Exit time). $T_{i}^{\text {exit }}$, the time before the semi-Markov process exits state $i$, follows an exponential distribution with rate $\sum_{\substack{j=1 \\ j \neq i}}^{N} \lambda_{i j} p_{i j}^{\text {forw }}$, where $p_{i j}^{\text {forw }}$ represents the probability that node $i$ hands over the message to node $j$ according to the forwarding scheme in use. $T_{i}^{\text {exit }}$ 's expected value is thus given by the following:

$$
E\left[T_{i}^{e x i t}\right]=\frac{1}{\sum_{\substack{j=1 \\ j \neq i}}^{N} \lambda_{i j} p_{i j}^{f o r w}} .
$$

Proof. See [3].

Below we derive the transition probabilities associated with the embedded chain in Figure 1 for each of the forwarding schemes described in Section 3.2

Proposition 1 (General form of the transition matrix for the forwarding process). The transition matrix associated with the process of forwarding a message from a source node $i$ to the destination node $d$ is given in Equation 5. where, as an example, $d=N$.

$$
\mathbf{P}=\left(\begin{array}{ccccc}
0 & p_{12} & \ldots & p_{1, N-1} & p_{1, N} \\
p_{21} & 0 & \ldots & p_{2, N-1} & p_{2, N} \\
\vdots & \vdots & \ddots & \vdots & \vdots \\
\vdots & \vdots & \vdots & \ddots & \vdots \\
0 & 0 & \ldots & 0 & 1
\end{array}\right)
$$

The state associated with the destination node $d$ is absorbing, because in state $d$ the forwarding process is completed.

Theorem 2 (Transition probabilities $p_{i j}$ ). Probabilities $p_{i j}$ in Equation 5 are given by:

$$
p_{i j}=\frac{\lambda_{i j} p_{i j}^{f o r w}}{\sum_{z} \lambda_{i z} p_{i z}^{f o r w}},
$$

where $\lambda_{i j}$ denotes the rate of encounters between node $i$ and node $j$, and $p_{i j}^{\text {forw }}$ represents the probability that node $i$ hands over the message to node $j$ according to the forwarding scheme in use.

\footnotetext{
${ }^{2}$ The chain is different for different destinations, because the convenient relays are generally not the same. However, for the sake of readability, in the following we drop superscript $d$.
} 
Proof. See [3].

Both exit time $T_{i}^{\text {exit }}$ and transition probabilities $p_{i j}$ are dependent on $p_{i j}^{\text {forw }}$. Thus, in the following we derive $p_{i j}^{\text {forw }}$ for each of the reference forwarding policies in Section 3.2.

Lemma 1 ( $p_{i j}^{\text {forw }}$ for Direct Transmission). The probability $p_{i j}^{\text {forw }}$ that node $i$ hands over the message to node $j$ when the Direct Transmission policy is in use is given by the following:

Proof. See [3].

$$
p_{i j}^{\text {forw }}=\left\{\begin{array}{l}
1 j=D \\
0 \text { otherwise }
\end{array}\right.
$$

Lemma 2 ( $p_{i j}^{\text {forw }}$ for Always Forward). The probability $p_{i j}^{\text {forw }}$ that node $i$ hands over the message to node $j$ when the Always Forward policy is in use is given by the following:

$$
p_{i j}^{\text {forw }}=1, \quad \forall i, j
$$

Proof. See [3].

Lemma 3 ( $p_{i j}^{\text {forw }}$ for Direct Acquaintance and Social Forwarding). Under the Direct Acquaintance strategy, the probability $p_{i j}^{\text {forw }}$ that node $i$ hands over the message to node $j$ can be computed as:

$$
p_{i j}^{\text {forw }}= \begin{cases}1 & F_{i, d}^{D A}<F_{j, d}^{D A} \\ 0 & \text { otherwise }\end{cases}
$$

Analogously, for the Social Forwarding scheme we have for $p_{i j}^{\text {forw }}$ :

$$
p_{i j}^{\text {forw }}= \begin{cases}1 & F_{i, d}^{S F}<F_{j, d}^{S F} \\ 0 & \text { otherwise }\end{cases}
$$

Fitness $F_{i, j}^{D A}$ and $F_{i, j}^{S F}$ are defined in Equations 11 and .

Proof. See 3 .

Theorems 1 and 2 completely define the forwarding Markov process. Thus, we can exploit well known algorithms for Markov chain transient analysis in order to compute significant properties of the forwarding process. In the following, we describe how to compute the expected delay and the expected number of hops travelled by messages.

Theorem 3 (Expected delay). The expected delay $E\left[D_{i}^{d}\right]$ for a message generated by node $i$ and addressed to node $d$ can be obtained from the minimal non-negative solution to the following system:

$$
\left\{\begin{array}{l}
E\left[D_{i}^{d}\right]=0 \quad i=d \\
E\left[D_{i}^{d}\right]=E\left[T_{i}^{e x i t}\right]+\sum_{j \neq d} p_{i j} E\left[D_{j}^{d}\right] \forall i \neq d
\end{array}\right.
$$

Proof. See [3]. 
Theorem 4 (Expected number of hops). The expected number of hops $E\left[H_{i}^{d}\right]$ travelled by a message generated by node $i$ and addressed to node $d$ can be obtained from the minimal non-negative solution to the following system:

$$
\left\{\begin{array}{l}
E\left[H_{i}^{d}\right]=0 \quad i=d \\
E\left[H_{i}^{d}\right]=1+\sum_{j \neq d} p_{i j} E\left[H_{j}^{d}\right] \forall i \neq d
\end{array}\right.
$$

Proof. See 3 .

\section{Performance Evaluation of Social-Aware Forwarding}

In this section we provide a detailed analysis of the performance of the Direct Transmission, Always Forward, Direct Acquaintance, and Social Forwarding schemes using the analytical model that we have described above. Under the assumptions in Section 3.1. this model is exact (for a comparison between analytical and simulation results please refer to [3]).

In the following we consider 15 nodes, which move around in the network and exchange messages according to the policies defined in Section 3.2. We assume that node movements are triggered by their social relationships with the other nodes of the network. Each scenario we consider is characterized by a different social structure connecting the nodes of the network. Based on this structure, we define node mobility according to the following algorithm. We assume that the default meeting rate is $\lambda$ for each pair of nodes connected by a social link. For those scenarios in which nodes are grouped into communities, however, assuming the user is in touch with $n$ communities, the rate of contact with users in each of those communities is $\lambda / n$.

Solving the systems of equations in Theorems 3 and 4 provides us with a $N \times N$ matrix for the expected delay and a $N \times N$ matrix for the expected number of hops. Thus, the entry at position $(i, j)$ in the matrix gives the expected delay (or number of hops) value for the $i-j$ node pair. For ease of visualization, we rely on a histogram of the expected delay and of the expected number of hops computed for the $N(N-1)$ pairs of interests. The bin width is set to 2 for the histograms of the expected delay and to 1 for the expected number of hops. Finally, please note that, in all the cases analyzed below, the resulting expected delay between any pair of nodes is a function of $\lambda$. In order to be able to plot such results we set $\lambda$ to 1 . This choice has absolutely no effect on our performance comparison, because $\lambda$ appears only as a multiplying factor.

\subsection{Homogeneous Network}

Let us start our performance evaluation with the case of a complete social graph, i.e., a graph in which an edge connecting any pair of nodes exists. With this configuration all nodes are homogeneous from a mobility standpoint, i.e., every pair of nodes meets at the same rate $\lambda$. As a consequence, the concept of community does not apply here. 
From Theorem 3, we obtain that the expected delay experienced by messages is the same for all the four policies and equal to $\frac{1}{\lambda}$. This result is not surprising, since all nodes are equivalent in this configuration, and choosing the one or the other does not make any difference. However, the different forwarding strategies may drastically differ in the number of hops needed to bring the message to its destination. Indeed, Table 1 shows that the Direct Transmission, Direct Acquaintance, and Social Forwarding schemes are all able to detect the fact that, as all nodes are equally good as relays, the most convenient strategy is to appoint the source of the message as its unique forwarder. Instead, the Always Forward scheme, which continuously delegates the forwarding of the message to any new encounter, needs much more relays (from which the high number of hops), which in turn imply many (unneeded) transmissions, with the consequence of poor resource utilization.

Table 1. Expected number of hops

\begin{tabular}{ccccc} 
& DT & AF & DA & SF \\
\hline \# hops & 1 & 14 & 1 & 1
\end{tabular}

This homogeneous scenario is the one commonly used to evaluate the Epidemic forwarding strategy 21, which under ideal conditions (i.e., infinite bandwidth, infinite buffer space on devices, infinite battery lifetime, no contention, etc.) is the optimal forward policy as far as the expected delay is concerned. Being a multi-copy strategy, the Epidemic protocol does not fit into our model. However, we can exploit results presented in 22 in order to compare our singlecopy strategies with Epidemic routing. The expected delay $E\left[D^{e p i}\right]$ under Epidemic routing converges to $\frac{\ln N}{\beta(N-1)}$ as $N \rightarrow \infty$. This value is thus generally much smaller than $\frac{1}{\lambda}$, and it decreases as $N$ increases. However, the price to pay for this quick delivery is in terms of the number of copies disseminated into the network. According to 22 , the expected number of copies $E\left[C^{e p i}\right]$ injected into the network by Epidemic routing is $\frac{N-1}{2}$. As $N$ increases, $E\left[C^{e p i}\right]$ also increases, thus flooding the network with many copies of the same data. When ideal conditions assumption is released, this will drastically affect the performance of Epidemic routing, and the delay provided will be much smaller than the optimal value, as shown in [1].

\subsection{Connected Communities}

While in the homogeneous case all nodes were equal as far as their meetings were concerned, here we consider the case of a heterogeneous network. We equally distribute our 15 nodes into 3 communities, namely, $C 1, C 2$, and $C 3$. Each community is a complete subgraph, meaning that all nodes within each community are connected with each other. We also add links between communities in the social graph. These links are edges connecting a node in one community to another node in another community. We hereafter refer to the nodes having inter-community links as travellers. We assign travellers only to community $C 1$, which makes the network still connected (i.e., it exists at least one multi-hop 
path between every pair of nodes). However, with this configuration, community $C 2$ and $C 3$ cannot communicate directly, and they have to exploit the forwarding capabilities of the visiting travellers from $C 1$. In the following, for ease of reading, we denote with indices from 1 to 5 the nodes in the first community $(C 1)$, with indices from 6 to 10 the nodes in the community $(C 2)$, and so on.

Figure 2 shows the expected delay experienced by messages in this scenario. Both the Direct Acquaintance and the Direct Transmission schemes are not able to deliver a subset of messages. The Direct Transmission scheme suffers when the source and the destination of the message do not get in touch with each other directly, thus producing in this case infinite delays. This is because with Direct Transmission nodes can only deliver their messages directly to the destination, thus missing all the opportunities offered by relaying: when the destination is never met, the message cannot be delivered. With Direct Acquaintance a node hands over a message to a node that has a higher probability of meeting the destination, measured in terms of direct encounters (Equation 1). The traveler that visits $C 1$ does not meet any nodes of $C 3$ directly, thus it is not considered a good relay by the Direct Acquaintance scheme. A more efficient strategy should also consider the transitivity of opportunities (e.g., node $a$ meets $b$, which in turn meets $c$, thus $a$ can be considered a good relay for destination $c$ ). This transitivity of encounters is detected by the Social Forwarding strategy, which indeed is able to deliver all messages to their destinations. The Always Forward strategy is also able to deliver all messages, but using many relays (Figure 3). The reason is that, being the forwarding opportunities so limited, with the Always Forward strategy the destination is typically found by chance after many (bad) relays have been used.
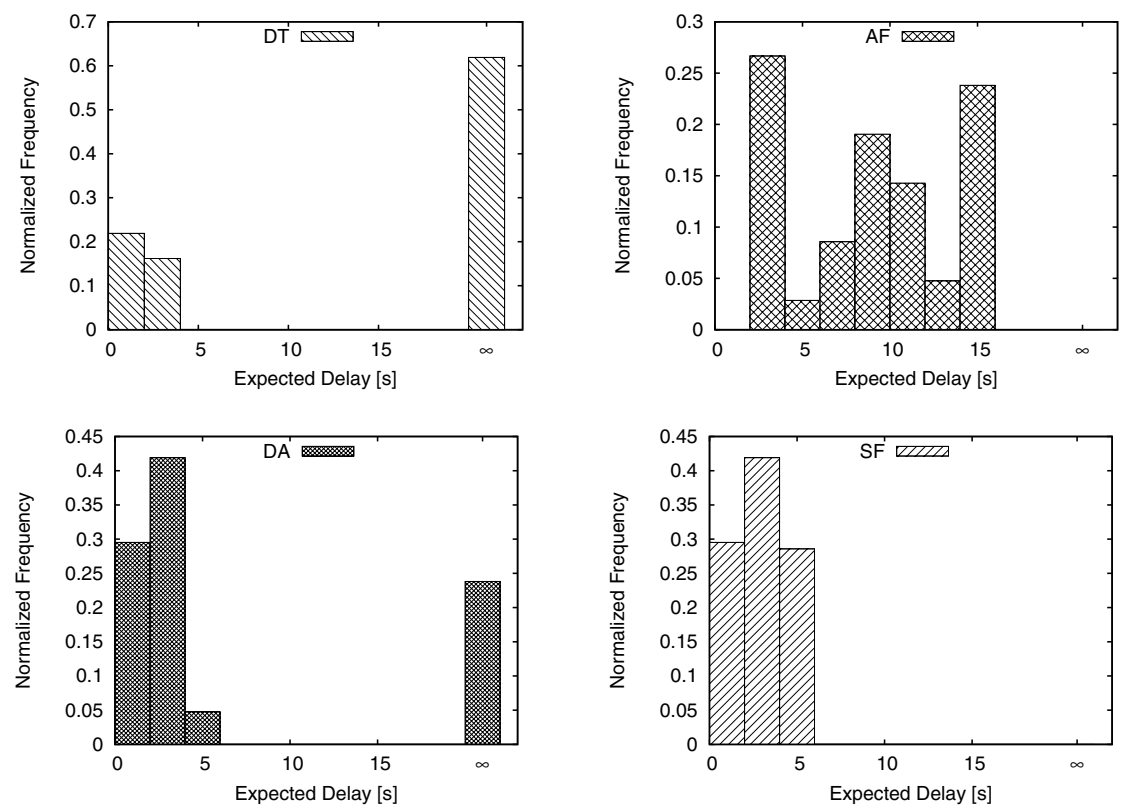

Fig. 2. Expected delay with connected communities (Sec. 4.2) 

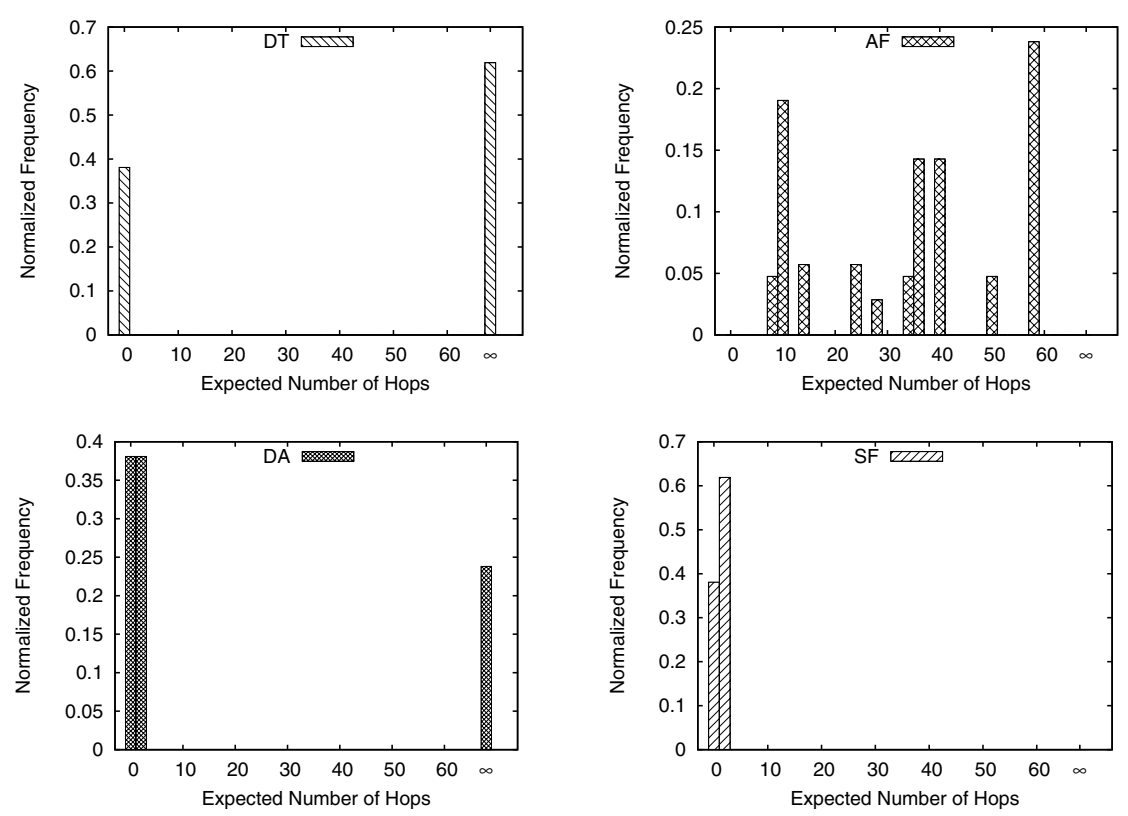

Fig. 3. Expected number of hops with connected communities (Sec. 4.2)

\section{Conclusions}

In this paper we have proposed an analytical model based on Markov processes for social-aware forwarding in opportunistic networks. Using this model, we have discussed how to compute the expected delay and the expected number of hops of messages delivered according to four reference forwarding schemes, of which two are able to exploit social information when making forwarding decisions. In the second part of the paper, we have used the model to compare the forwarding performance of social-oblivious and social-aware strategies in terms of expected delay and expected number of hops. In general, social-aware policies turn out to provide lower delays while at the same time keeping the number of hops down, thus improving the efficiency of the network. We have also shown how the ability of exploiting indirect connections between nodes may be a key strategy when forwarding opportunities are limited, and for this reason we have identified the Social Forwarding strategy as the most promising social-aware approach.

Acknowledgments. This work was partially funded by the European Commission under the SOCIALNETS (217141) FET-PERADA project.

\section{References}

1. Boldrini, C., Conti, M., Passarella, A.: Impact of social mobility on routing protocols for opportunistic networks. In: Proceedings of IEEE WoWMoM 2007. pp. 1-6 (2007) 
2. Boldrini, C., Conti, M., Passarella, A.: Exploiting users' social relations to forward data in opportunistic networks: The HiBOp solution. Pervasive and Mobile Computing 4(5), 633-657 (2008)

3. Boldrini, C., Conti, M., Passarella, A.: Modelling social-aware forwarding in opportunistic networks. Tech. rep., IIT-CNR (2010), http://bruno1.iit.cnr.it/ chiara/perform10_tr.pdf

4. Burleigh, S., Hooke, A., Torgerson, L., Fall, K., Cerf, V., Durst, B., Scott, K., Weiss, H.: Delay-tolerant networking: an approach to interplanetary internet. IEEE Communications Magazine 41(6), 128-136 (2003)

5. Daly, E., Haahr, M.: Social network analysis for information flow in disconnected Delay-Tolerant MANETs. IEEE Transactions on Mobile Computing pp. 606-621 (2008)

6. Fall, K., Farrell, S.: DTN: an architectural retrospective. IEEE Journal on Selected Areas in Communications 26(5), 828 (2008)

7. González, M., Hidalgo, C., Barabási, A.: Understanding individual human mobility patterns. Nature 453(7196), 779-782 (2008)

8. Groenevelt, R., Nain, P., Koole, G.: The message delay in mobile ad hoc networks. Performance Evaluation 62(1-4), 210-228 (2005)

9. Grossglauser, M., Tse, D.: Mobility increases the capacity of ad hoc wireless networks. IEEE/ACM Transactions on Networking 10(4), 477-486 (2002)

10. Haas, Z., Small, T.: A new networking model for biological applications of ad hoc sensor networks. IEEE/ACM Transactions on Networking 14(1), 27-40 (2006)

11. Hui, P., Crowcroft, J., Yoneki, E.: Bubble rap: social-based forwarding in delay tolerant networks. In: Proceedings of the 9th ACM MobiHoc. pp. 241-250. ACM (2008)

12. Karagiannis, T., Le Boudec, J.Y., Vojnović, M.: Power law and exponential decay of inter contact times between mobile devices. In: Proceedings of the 13th ACM MobiCom '07. pp. 183-194 (2007)

13. Lindgren, A., Doria, A., Schelén, O.: Probabilistic routing in intermittently connected networks. LNCS pp. 239-254 (2004)

14. Ott, J.: Application protocol design considerations for a mobile internet. In: Proceedings of first ACM/IEEE international workshop on Mobility in the evolving internet architecture. p. 80. ACM (2006)

15. Ross, S.: Introduction to probability models. Academic Press (2007)

16. Scott, J., Hui, P., Crowcroft, J., Diot, C.: Haggle: A networking architecture designed around mobile users. In: Proceedings of IFIP WONS (2006)

17. Silvis, J., Niemeier, D., D'Souza, R.: Social networks and travel behavior: Report from an integrated travel diary. In: 11th International Conference on Travel Behaviour Reserach, Kyoto (2006)

18. Spyropoulos, T., Psounis, K., Raghavendra, C.: Efficient routing in intermittently connected mobile networks: The multiple-copy case. IEEE/ACM Transactions on Networking 16(1), 77-90 (2008)

19. Spyropoulos, T., Psounis, K., Raghavendra, C.: Efficient routing in intermittently connected mobile networks: The single case. IEEE/ACM Transactions on Networking 16(1), 63-76 (2008)

20. Spyropoulos, T., Turletti, T., Obraczka, K.: Routing in Delay-Tolerant Networks Comprising Heterogeneous Node Populations. IEEE Transactions on Mobile Computing pp. 1132-1147 (2009)

21. Vahdat, A., Becker, D.: Epidemic routing for partially connected ad hoc networks. Tech. Rep. CS-2000-06, Duke University (2000)

22. Zhang, X., Neglia, G., Kurose, J., Towsley, D.: Performance modeling of epidemic routing. Computer Networks 51(10), 2867-2891 (2007) 